\title{
An Overview of Cognitive Remediation Therapy for People with Severe Mental Illness
}

\author{
Cherrie Galletly ${ }^{1,2,3}$ and Ashlee Rigby ${ }^{2,3}$ \\ ${ }^{1}$ Adelaide Health Service, Northern Mental Health Directorate, Department of Health and Ageing, \\ Adelaide, SA 5000, Australia \\ ${ }^{2}$ Mental Health Services (SA), Ramsay Health Care, The Adelaide Clinic, Adelaide, SA 5082, Australia \\ ${ }^{3}$ Discipline of Psychiatry, School of Medicine, The University of Adelaide, Adelaide, SA 5000, Australia
}

Correspondence should be addressed to Cherrie Galletly; cherrie.galletly@adelaide.edu.au

Received 14 December 2012; Accepted 1 January 2013

Academic Editors: K. Hashimoto, K.-H. Lin, and M. Schmitter-Edgecombe

Copyright (C) 2013 C. Galletly and A. Rigby. This is an open access article distributed under the Creative Commons Attribution License, which permits unrestricted use, distribution, and reproduction in any medium, provided the original work is properly cited.

Cognitive remediation refers to nonpharmacological methods of improving cognitive function in people with severe mental disorders. Cognitive remediation therapy (CRT) can be delivered via computerised programs, of varying length and complexity, or can be undertaken one-on-one by a trained clinician. There has been a considerable interest in cognitive remediation, driven by recognition that cognitive deficits are a major determinant of outcome in people with severe, chronic mental illnesses. CRT has been shown to be effective, especially if combined with vocational rehabilitation.

\section{Cognitive Impairment in Severe Mental Illness}

The term "severe mental illness" (SMI) is generally taken to encompass chronic psychotic disorders. Whilst many people in this category suffer from schizophrenia, disorders such as schizoaffective disorder, delusional disorder and bipolar disorder are also commonly associated with persistent symptoms, cognitive impairment and long-term disability [1]. Data collected from 1875 people with SMI demonstrated that their current cognitive ability was markedly impaired, compared to the general population. On average, people with psychosis scored 1.6 standard deviations below the general population on a test of information processing speed [1].

There are some differences between diagnostic groups. Children who later develop schizophrenia are more likely to be slow to learn to speak [2] and to have impaired language and arithmetic ability during childhood [3], reflecting neurodevelopmental processes underlying adult schizophrenia [4]. In contrast, people with bipolar disorder tend to have average or superior educational achievement as children, suggesting a different illness trajectory [5]. Nevertheless, despite premorbid differences, cognitive impairment is evident in both affective psychoses (bipolar disorder and psychotic depression) and nonaffective psychoses (schizophrenia) [6] even in the early stages of the disorder. Cognitive impairment is generally most profound and pervasive in schizophrenia [7].

Vocational outcomes are poor in people with SMI, with many experiencing long-term unemployment and welfare dependence, despite evidence that they could gain considerable benefit from engagement in paid employment [8]. Numerous studies have demonstrated an association between the severity of cognitive impairment and functional, social, and occupational outcomes in schizophrenia $[9,10]$. Similarly, in bipolar disorder, cognitive deficits have been shown to be associated with reduced psychosocial function [11, 12]. Cognitive impairment is an important determinant of poor engagement with vocational rehabilitation services [13] and failure to maintain job tenure [14]. Research into measures to improve cognition is therefore driven by the hope that such improvements might lead to better long-term economic as well as functional outcomes, along with greater opportunities for social inclusion. 
A number of medications have been trialled, specifically targeting cognitive function [15]. Treatment with clozapine is associated with improvements in working memory although normal levels of performance are not achieved [16]. Whilst other atypical antipsychotic drugs have been shown to be associated with improved cognition, it appears that much of this improvement is an artefact of study design with failure to properly address practice effects [17].

Very high levels of tobacco use are found amongst people with schizophrenia $[18,19]$, and interestingly nicotine improves their ability to filter out irrelevant information and to manage complex environments [20]. Drugs acting on nicotinic receptors may therefore have a role in enhancing cognition in schizophrenia. A number of other targets for drug development have been identified, but so far none of the drugs available have shown substantial benefit in clinical trials [15]. This lack of progress in psychopharmacology has highlighted the need for effective nonpharmacological methods of cognitive remediation.

\section{Cognitive Remediation: Study Populations}

Virtually all of the research regarding cognitive remediation in SMI has been undertaken in people with schizophrenia, perhaps because, as described above, schizophrenia has been shown to be associated with the most severe cognitive deficits. There has been one study comparing cognitive remediation in schizophrenia and schizoaffective disorder [21] which found that cognitive enhancement therapy, a two-year intervention including both computer training and social cognitive group sessions, was equally effective in both groups. Harvey et al. (2010) suggest that people with bipolar disorder might also benefit from the treatments used to address cognitive deficits in schizophrenia [22]. Deckersbach et al. (2010) have reported that a 14-session cognitive remediation program designed to treat both residual depressive symptoms and cognitive impairment in people with bipolar disorder, and delivered to each participant individually, was effective in reducing depression and improving occupational and psychosocial functioning. This was a small study $(N=18)$ with no control group, but it does provide an indication that further research into cognitive remediation in bipolar disorder is warranted [23].

\section{Methods of Cognitive Remediation}

An expert consensus group has defined cognitive remediation Therapy (CRT) as "a behavioural-training based intervention that aims to improve cognitive processes (attention, memory, executive function, social cognition, or metacognition) with the goal of durability and generalization" [24]. A variety of different methods of cognitive remediation have been described in the research literature. The starting point for this research was to establish whether it is possible to achieve any improvement in cognitive function in schizophrenia using non-pharmacological methods. Some of the earliest studies demonstrated that people with schizophrenia could be taught to improve their performance on the
Wisconsin Card Sorting Test $[25,26]$. Simple strategies such as vocalisation (speaking aloud whilst performing the task) have been shown to improve performance on complex tasks [27]. In other early studies, computer programs developed to retrain attentional deficits due to closed-head injury were used with people with schizophrenia. Results were mixed, with some studies reporting improvement [28, 29] while others found no change $[24,30]$.

Next, programs were developed to target the cognitive deficits characteristic of schizophrenia. These programs differ considerably in their theoretical underpinnings, methods, duration, and target outcomes. CRT programs generally take one of two approaches, either rehearsal learning, also termed drill and practice, or strategy coaching, where participants are helped to develop effective strategies to undertake cognitive tasks. Combinations of these two approaches can be utilised [31].

Whilst earlier studies often used clinician-administered CRT, recent studies most commonly utilise computerised CRT. Computerised methods have the advantages of being standardised and more efficient. Less staff time is needed as one staff member can supervise several participants, and staff do not require specialised skills. The larger and more successful studies have used programs such as Cogpack [32] and CogRehab [31]. Cogpack and Cogrehab consist of a series of computer-based programs which address a range of cognitive functions. Cognitive domains including memory, attention, visual information processing, language, and motor function are targeted using interactive training programs. Genevsky et al. (2010) randomly assigned participants to either 50 hours of playing computer games, or to 50 hours of computerised training of auditory perception and aural speech reception for 10 weeks. These exercises were used as it is thought that early auditory processing deficits are associated with impaired verbal memory, reading ability and emotional recognition. Subjects who undertook this training showed improvements in working memory, verbal learning and memory, and global cognition compared to the comparison group [33]. These programs allow therapy to be extended through independent exercise on a home computer. Computer-delivered programs are generally acceptable to participants, especially younger people for whom these programs resemble recreational computer games. Some studies use a combination of the two methods; for example, Royer et al. (2012) describe a combination of psychologist-assisted pencil and paper exercises and a computerised training program [34].

\section{Outcome Measures}

There is a considerable variation in outcome measures, and this makes comparison between studies difficult. The National Institute of Mental Health (NIMH) MATRICS (Measurement and Treatment Research to Improve Cognition in Schizophrenia) initiative attempted to address the lack of standardization of instruments used to measure cognition in schizophrenia. A consensus neuropsychological battery of 10 tests $[35,36]$ was developed to evaluate performance across seven domains previously shown to be impaired in schizophrenia. These domains are speed of processing, 
attention/vigilance, working memory, verbal learning, visual learning; reasoning and problem solving; and social cognition. Normative data for the MATRICS test battery has been obtained on 300 adults [35]. However, despite the availability of the MATRICS battery, a wide range of outcome measures continue to be used in research evaluating CRT [36].

\section{Efficacy of CRT}

Wykes et al. (2007) described a study using a cliniciandelivered CRT program in young people with schizophrenia. The program required 40 hours of individual work with each patient [37]. Tasks were designed to improve information processing strategies and included memory, complex planning, and problem solving. Three cognitive outcome measures were selected, and comparisons between the CRT group and the control group found statistically significant improvement in only one of these measures, with no significant effect of CRT on symptom severity or social functioning. There was some controversy about whether such modest gains are worth the investment of time and expertise [38].

However, in a meta-analysis of 40 studies of cognitive remediation in schizophrenia, Wykes et al. (2011) found that CRT resulted in significant improvements in global cognition with a mean effect size of 0.45 . Nearly all cognitive domains, including attention and vigilance, speed of processing, working memory, reasoning, problem solving, and social cognition demonstrated significant effect sizes ranging from 0.25 to 0.65 . Importantly, the effects on global cognition were sustained 3-12 months after CRT was completed (effect size $=0.43$ ). The average length of treatment was 32.3 hours provided across 16.7 weeks; however the sample size in most studies was small, ranging from 10 to 145 people. Wykes et al. (2011) concluded that CRT can provide benefits to patients with cognitive difficulties independent of therapy characteristics such as individual versus group therapy, patient characteristics or sample size [39].

Wykes et al. (2011) also proposed that nearly all cognitive domains respond to remediation in the treatment of schizophrenia. As cognitive remediation is not domain specific they propose that the outcome measure of any cognitive remediation therapy should be the effect it has on community functioning [39].

\section{Real-World Applications of CRT}

Improving performance on cognitive tests is not in itself of benefit; the goals of CRT are to improve real-world outcomes such as work, socialisation, and independent living skills. One of the shortcomings in CRT research has been that this therapy has often been provided without concurrent rehabilitation programs, and the more successful studies do combine CRT and psychosocial and/or vocational rehabilitation. For example, McGurk and Meltzer (2000) found that a combination of computer-aided CRT and vocational rehabilitation was more successful in returning people to work than vocational rehabilitation alone [32]. A meta-analysis by McGurk et al. (2007) reported that studies that included adjunctive psychiatric rehabilitation had significantly better outcomes, compared with cognitive remediation alone [40]. This makes sense as participants have the opportunity to apply their improved cognitive skills directly to the challenges of the workplace, resulting in iterative learning opportunities.

This is supported by a meta-analysis that concluded that CRT was more effective when it was provided with other adjunctive psychiatric rehabilitation [39]. In the short term, the CRT programs which focussed solely on cognitive skills had a small to medium effect size but demonstrated no significant difference at followup assessment [39]. These results indicate that in order to maximise the benefits of CRT, participants should be working or be involved in employmentfocused rehabilitation. This important finding, reported more than a decade ago, has nevertheless not been widely translated into services for people with SMI.

CRT has also been shown to improve symptoms in schizophrenia. Participants in a study by Lecardeur et al. (2009) received 2 novel CRTs, one targeting mental state attribution, and the other, mental flexibility, and compared both groups with a control group given treatment as usual. Symptoms decreased after therapy, but not in the control group. The mental flexibility group showed the most improvement [41]. Additionally, this study reported that participants described a subjective reduction of their cognitive deficits.

On the whole, CRT has been shown to have moderate improvements on global cognition and to a lesser extent functioning. However, greater improvement in functional outcomes can be achieved by combining psychosocial rehabilitation and CRT [40].

\section{Remediation of Social Cognitive Deficits}

Social cognition is a domain of cognitive function that includes the ability to understand and manage social interactions. Social cognition refers to aspects of cognition that are critical for social functioning and interpersonal relationships, such as accurate perception of the emotions of others, the ability to recognize important social cues, theory of mind (TOM), and the management of emotions in social situations [42]. Various components of social cognition have been found to be impaired in people with schizophrenia $[43,44]$. Lindenmayer et al. (2012) concluded that CRT combined with emotion perception remediation produced greater improvements in emotion recognition, emotion discrimination, social functioning, and neurocognition compared with CRT alone in chronic schizophrenia [45]. Furthermore, as has been shown previously in cognitive remediation research, training in one area of cognition can generalise to other social cognition domains which were not the primary focus of treatment [46]. Penadés et al. (2010) reported that improvement in executive functioning predicted improved daily functioning and social cognition among people with schizophrenia who had negative symptoms and neuropsychological impairment [47]. This provides further support for the generalizability of CRT training to a variety of cognitive domains. A metaanalysis of computerised CRT found a significant medium effect size of 0.64 (CI 0.29-0.99) for Social Cognition [48]. 


\section{Remediation of Cognitive Biases}

Cognitive illusions or biases are perceptions or judgements that deviate from reality in a systematic way and reflect inherent thinking errors made in processing information. Metacognitive training (MCT) has been developed as a treatment for people with schizophrenia to address cognitive biases thought to contribute to delusions [49]. MCT is a combination of psychoeducation, cognitive remediation, and cognitive-behavioural therapy (CBT). CBT is a focussed approach to therapy that helps individuals to identify unhelpful thoughts or behaviours and to learn healthy thought patterns and habits. By contrast, CRT is conceptualised as a behavioural-based training program for various areas of neurocognition and executive functioning which has generalizable effects on functioning. By combining these two therapeutic approaches MCT educates people about their cognitive biases and teaches them strategies to deal with biases which may impact on functioning [49].

MCT has been shown to be effective in retraining the "jumping to conclusions" bias and in theory of mind [50]. Balzan et al. (in press) enrolled 24 participants with schizophrenia and a history of delusions to a metacognitive training program. In one-hour individual sessions, participants were encouraged to think about the cognitive underpinnings of delusional ideation and to consider that their delusions may be maintained by a hypersalience of evidencehypothesis matches [51]. Hypersalience of evidence-hypothesis matches is one type of "jumping to conclusions" bias, where people make strong connections between existing beliefs and weak evidence [51, 52]. After two weeks of MCT, the severity of the delusions, and the extent of delusional preoccupation and conviction, had decreased compared to a treatment as usual comparison group [51].

\section{The Future of CRT}

As yet, CRT has not been commonly adopted in clinical practice. Wykes, writing in Nature (2010), proposes that the future of CRT lies in a more personalised approach. She suggests that research participants should be stratified, perhaps according to age or learning style, to identify the methods that are most effective according to these individual factors [38]. The goal would be to tailor an optimal remediation program for each individual. Ultimately, biological research methods utilising biomarkers or brain imaging might have a role in investigating the effects of CRT on brain function, and eventually in matching the treatment approach to the individual patient.

Clinically the future of CRT most likely lies in developing effective programs that combine CRT and psychosocial and vocational rehabilitation. Although the outcome measures differ, comparison between the results of clinician-delivered programs [37] and computerised CRT programs suggest that the computerised programs are generally equally effective [48]. Computerised programs are more likely to be adopted in clinical practice, with clinician-delivered programs used primarily for research. CRT programs would need to be standardised with built in measures of efficacy, so that they easily set up in community mental health services, require minimal staff supervision, and demonstrate sufficient improvement to justify the ongoing commitment of resources. Future research in this area should continue to look at the optimal modality for CRT and the effects on particular domains of cognition as well as evaluating the impact on general functional outcome.

\section{References}

[1] V. A. Morgan, A. Waterreus, and A. Jablensky, "People living with psychotic illness 2010," Report on the Second Australian National Survey, Australian Government Department of Health and Ageing, Canberra, Australia, 2011.

[2] P. Jones, B. Rodgers, R. Murray, and M. Marmot, "Child developmental risk factors for adult schizophrenia in the British 1946 birth cohort," The Lancet, vol. 344, no. 8934, pp. 1398-1402, 1994.

[3] D. J. Done, T. J. Crow, E. C. Johnstone, and A. Sacker, "Childhood antecedents of schizophrenia and affective illness: social adjustment at ages 7 and 11," British Medical Journal, vol. 309, no. 6956, pp. 699-703, 1994.

[4] R. M. Murray, P. Sham, J. Van Os, J. Zanelli, M. Cannon, and C. McDonald, "A developmental model for similarities and dissimilarities between schizophrenia and bipolar disorder," Schizophrenia Research, vol. 71, no. 2-3, pp. 405-416, 2004.

[5] T. Toulopoulou, S. Quraishi, C. McDonald, and R. M. Murray, "The Maudsley Family study: premorbid and current general intellectual function levels in familial bipolar I disorder and schizophrenia," Journal of Clinical and Experimental Neuropsychology, vol. 28, no. 2, pp. 243-259, 2006.

[6] S. K. Hill, J. L. Reilly, M. S. H. Harris et al., "A comparison of neuropsychological dysfunction in first-episode psychosis patients with unipolar depression, bipolar disorder, and schizophrenia," Schizophrenia Research, vol. 113, no. 2-3, pp. 167-175, 2009.

[7] J. Zanelli, A. Reichenberg, K. Morgan et al., "Specific and generalized neuropsychological deficits: a comparison of patients with various first-episode psychosis presentations," American Journal of Psychiatry, vol. 167, no. 1, pp. 78-85, 2010.

[8] D. J. Martino, E. Marengo, A. Igoa et al., "Neurocognitive and symptomatic predictors of functional outcome in bipolar disorders: a prospective 1 year follow-up study," Journal of Affective Disorders, vol. 116, no. 1-2, pp. 37-42, 2009.

[9] M. Kukla, G. R. Bond, and H. Xie, "A prospective investigation of work and nonvocational outcomes in adults with severe mental illness," Journal of Nervous and Mental Disease, vol. 200, no. 3, pp. 214-222, 2012.

[10] M. F. Green, R. S. Kern, D. L. Braff, and J. Mintz, "Neurocognitive deficits and functional outcome in schizophrenia: are we measuring the "right stuff"?" Schizophrenia Bulletin, vol. 26, no. 1, pp. 119-136, 2000.

[11] R. S. E. Keefe, M. Poe, T. M. Walker, J. W. Kang, and P. D. Harvey, "The schizophrenia cognition rating scale: an interview-based assessment and its relationship to cognition, real-world functioning, and functional capacity," American Journal of Psychiatry, vol. 163, no. 3, pp. 426-432, 2006.

[12] B. Solé, C. M. Bonnin, C. Torrent et al., "Neurocognitive impairment and psychosocial functioning in bipolar II disorder," Acta Psychiatrica Scandinavica, vol. 125, no. 4, pp. 309-317, 2012.

[13] M. K. O’Connor, L. Mueller, A. van Ormer et al., "Cognitive impairment as barrier to engagement in vocational services 
among veterans with severe mental illness," Journal of Rehabilitation Research and Development, vol. 48, no. 5, pp. 597-607, 2011.

[14] J. M. Gold, R. W. Goldberg, S. W. McNary, L. B. Dixon, and A. F. Lehman, "Cognitive correlates of job tenure among patients with severe mental illness," American Journal of Psychiatry, vol. 159, no. 8, pp. 1395-1402, 2002.

[15] C. Galletly, "Recent advances in treating cognitive impairment in schizophrenia," Psychopharmacology, vol. 202, no. 1-3, pp. 259-273, 2009.

[16] C. A. Galletly, C. R. Clark, A. C. McFarlane, and D. L. Weber, "The effect of clozapine on the speed and accuracy of information processing in schizophrenia," Progress in NeuroPsychopharmacology and Biological Psychiatry, vol. 24, no. 8, pp. 1329-1338, 2000.

[17] A. Szöke, A. Trandafir, M.-E. Dupont, A. Méary, F. Schürhoff, and M. Leboyer, "Longitudinal studies of cognition in schizophrenia: meta-analysis," British Journal of Psychiatry, vol. 192, no. 4, pp. 248-257, 2008.

[18] J. De Leon and F. J. Diaz, "A meta-analysis of worldwide studies demonstrates an association between schizophrenia and tobacco smoking behaviors," Schizophrenia Research, vol. 76, no. 2-3, pp. 135-157, 2005.

[19] J. Cooper, S. G. Mancuso, R. Borland, T. Slade, C. Galletly, and D. Castle, "Tobacco smoking among people living with a psychotic illness: the second Australian survey of psychosis," Australian and New Zealand Journal of Psychiatry, vol. 46, no. 9, pp. 851-863, 2012.

[20] R. S. Barr, M. A. Culhane, L. E. Jubelt et al., "The effects of transdermal nicotine on cognition in nonsmokers with schizophrenia and nonpsychiatric controls," Neuropsychopharmacology, vol. 33, no. 3, pp. 480-490, 2008.

[21] K. E. Lewandowski, S. M. Eack, S. S. Hogarty, D. P. Greenwald, and M. S. Keshavan, "Is cognitive enhancement therapy equally effective for patients with schizophrenia and schizoaffective disorder?" Schizophrenia Research, vol. 125, no. 2-3, pp. 291-294, 2011.

[22] P. D. Harvey, A. P. Wingo, K. E. Burdick, and R. J. Baldessarini, "Cognition and disability in bipolar disorder: lessons from schizophrenia research," Bipolar Disorders, vol. 12, no. 4, pp. 364-375, 2010.

[23] T. Deckersbach, A. A. Nierenberg, R. Kessler et al., "Cognitive rehabilitation for bipolar disorder: an open trial for employed patients with residual depressive symptoms," CNS Neuroscience and Therapeutics, vol. 16, no. 5, pp. 298-307, 2010.

[24] R. H. B. Benedict, A. E. Harris, T. Markow, J. A. McCormick, K. H. Nuechterlein, and R. F. Asarnow, "Effects of attention training on information processing in schizophrenia," Schizophrenia Bulletin, vol. 20, no. 3, pp. 537-546, 1994.

[25] S. L. Rossell and A. S. David, "Improving performance on the WCST: variations on the original procedure. Wisconsin Card Sorting Test," Schizophrenia Research, vol. 28, no. 1, pp. 63-76, 1997.

[26] P. Stratta, F. Mancini, P. Mattei et al., "Remediation of Wisconsin Card Sorting Test performance in schizophrenia. A controlled study," Psychopathology, vol. 30, no. 2, pp. 59-66, 1997.

[27] K. E. Harvey, C. A. Galletly, C. Field, and M. Proeve, “The effects of verbalisation on cognitive performance in schizophrenia: a pilot study using tasks from the Delis Kaplan Executive Function System," Neuropsychological Rehabilitation, vol. 19, no. 5, pp. 733-741, 2009.
[28] M. Hermanutz and J. Gestrich, "Computer-assisted attention training in schizophrenics. A comparative study", European Archives of Psychiatry and Clinical Neuroscience, vol. 240, no. 45, pp. 282-287, 1991.

[29] A. Medalia, M. Aluma, W. Tryon, and A. E. Merriam, "Effectiveness of attention training in schizophrenia," Schizophrenia Bulletin, vol. 24, no. 1, pp. 147-152, 1998.

[30] C. D. Field, C. Galletly, D. Anderson, and P. Walker, "Computeraided cognitive rehabilitation: possible application to the attentional deficit of schizophrenia, a report of negative results," Perceptual and Motor Skills, vol. 85, no. 3, pp. 995-1002, 1997.

[31] G. E. Hogarty, S. Flesher, R. Ulrich et al., "Cognitive enhancement therapy for schizophrenia: effects of a 2-year randomized trial on cognition and behavior," Archives of General Psychiatry, vol. 61, no. 9, pp. 866-876, 2004.

[32] S. R. McGurk and H. Y. Meltzer, "The role of cognition in vocational functioning in schizophrenia," Schizophrenia Research, vol. 45, no. 3, pp. 175-184, 2000.

[33] A. Genevsky, C. T. Garrett, P. P. Alexander, and S. Vinogradov, "Cognitive training in schizophrenia: a neuroscience-based approach," Dialogues in Clinical Neuroscience, vol. 12, no. 3, pp. 416-421, 2010.

[34] A. Royer, A. Grosselin, C. Bellot et al., "Is there any impact of cognitive remediation on an ecological test in schizophrenia?" Cognitive Neuropsychiatry, vol. 17, no. 1, pp. 19-35, 2012.

[35] P. D. Harvey and B. A. Cornblatt, "Pharmacological treatment of cognition in schizophrenia: an idea whose method has come," American Journal of Psychiatry, vol. 165, no. 2, pp. 163-165, 2008.

[36] K. H. Nuechterlein, M. F. Green, R. S. Kern et al., "The MATRICS consensus cognitive battery, part 1: test selection, reliability, and validity," American Journal of Psychiatry, vol. 165, no. 2, pp. 203-213, 2008.

[37] T. Wykes, C. Reeder, S. Landau et al., "Cognitive remediation therapy in schizophrenia: randomised controlled trial," British Journal of Psychiatry, vol. 190, pp. 421-427, 2007.

[38] T. Wykes, "Cognitive remediation therapy needs funding," Nature, vol. 468, no. 7321, pp. 165-166, 2010.

[39] T. Wykes, V. Huddy, C. Cellard, S. R. McGurk, and P. Czobor, "A meta-analysis of cognitive remediation for schizophrenia: methodology and effect sizes," American Journal of Psychiatry, vol. 168, no. 5, pp. 472-485, 2011.

[40] S. R. McGurk, E. W. Twamley, D. I. Sitzer, G. J. McHugo, and K. T. Mueser, "A meta-analysis of cognitive remediation in schizophrenia," American Journal of Psychiatry, vol. 164, no. 12, pp. 1791-1802, 2007.

[41] L. Lecardeur, E. Stip, M. Giguere, G. Blouin, J. P. Rodriguez, and M. Champagne-Lavau, "Effects of cognitive remediation therapies on psychotic symptoms and cognitive complaints in patients with schizophrenia and related disorders: a randomized study," Schizophrenia Research, vol. 111, no. 1-3, pp. 153-158, 2009.

[42] M. F. Green, B. Olivier, J. N. Crawley, D. L. Penn, and S. Silverstein, "Social cognition in schizophrenia: recommendations from the MATRICS New Approaches Conference," Schizophrenia Bulletin, vol. 31, no. 4, pp. 882-887, 2005.

[43] E. Bora, M. Yucel, and C. Pantelis, "Theory of mind impairment in schizophrenia: meta-analysis," Schizophrenia Research, vol. 109, no. 1-3, pp. 1-9, 2009.

[44] P. Stratta, M. Bustini, E. Daneluzzo, I. Riccardi, M. D’Arcangelo, and A. Rossi, "Deconstructing theory of mind in schizophrenia," Psychiatry Research, vol. 190, no. 1, pp. 32-36, 2011. 
[45] J-P. Lindenmayer, S. R. McGurk, and A. Khan, "Improving social cognition in schizophrenia: a pilot intervention combining computerized social cognition training with cognitive remediation," Schizophrenia Bulletin. In press.

[46] W. Wölwer and N. Frommann, "Social-cognitive remediation in schizophrenia: generalization of effects of the training of affect recognition (TAR)," Schizophrenia Bulletin, vol. 37, supplement 2, pp. S63-S70, 2011.

[47] R. Penadés, R. Catalán, O. Puig et al., "Executive function needs to be targeted to improve social functioning with Cognitive Remediation Therapy (CRT) in schizophrenia," Psychiatry Research, vol. 177, no. 1-2, pp. 41-45, 2010.

[48] O. Grynszpan, S. Perbal, A. Pelissolo et al., "Efficacy and specificity of computer-assisted cognitive remediation in schizophrenia: a meta-analytical study," Psychological Medicine, vol. 41, no. 1, pp. 163-173, 2011.

[49] S. Moritz, F. Vitzthum, S. Randjbar, R. Veckenstedt, and T. S. Woodward, "Detecting and defusing cognitive traps: metacognitive intervention in schizophrenia," Current Opinion in Psychiatry, vol. 23, no. 6, pp. 561-569, 2010.

[50] T. S. Woodward, R. Mizrahi, M. Menon, and B. K. Christensen, "Correspondences between theory of mind, jumping to conclusions, neuropsychological measures and the symptoms of schizophrenia," Psychiatry Research, vol. 170, no. 2-3, pp. 119123, 2009 .

[51] R. Balzan, P. Delfabbro, C. Galletly, and T. Woodward, "Metacognitive training for patients with schizophrenia: preliminary evidence for a targeted single-module program," In Submission.

[52] R. Balzan, P. Delfabbro, C. Galletly, and T. Woodward, "Reasoning heuristics across the psychosis continuum: the contribution of hypersalient evidence-hypothesis matches," Cognitive Neuropsychiatry, vol. 17, no. 5, pp. 431-450, 2012. 


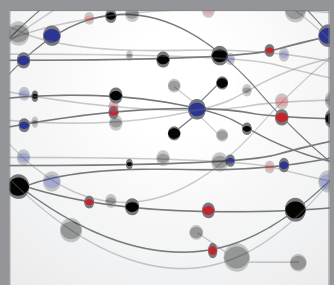

The Scientific World Journal
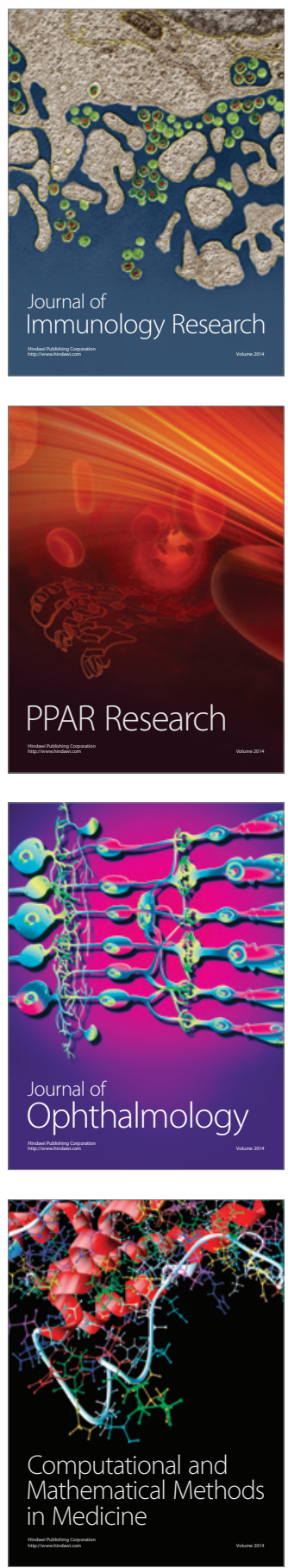

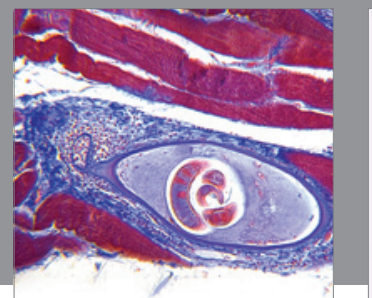

Gastroenterology

Research and Practice
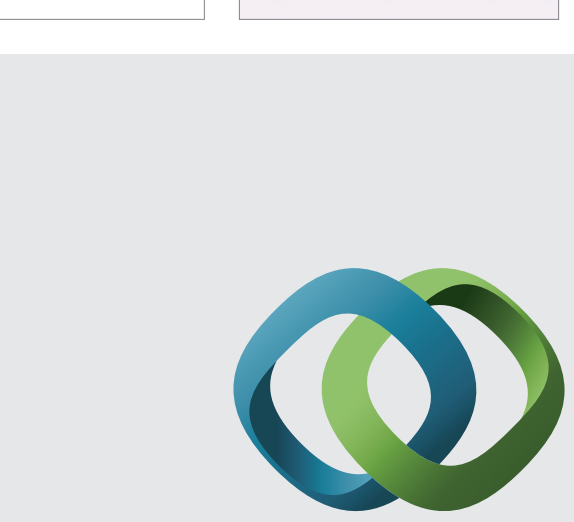

\section{Hindawi}

Submit your manuscripts at

http://www.hindawi.com
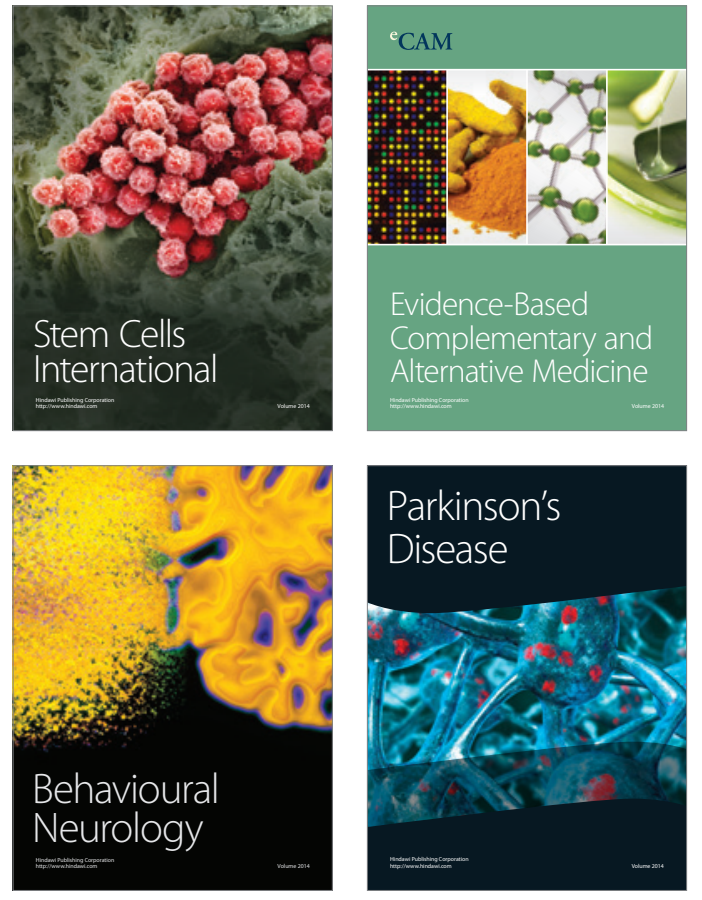
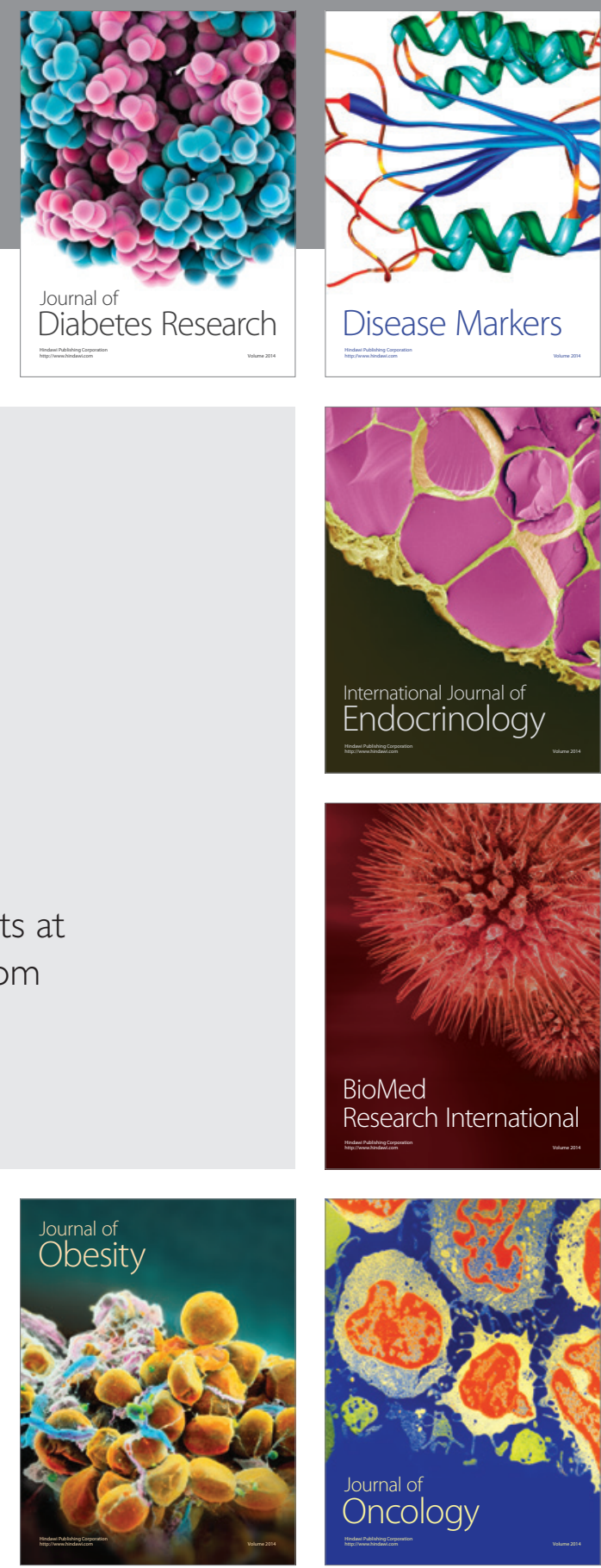

Disease Markers
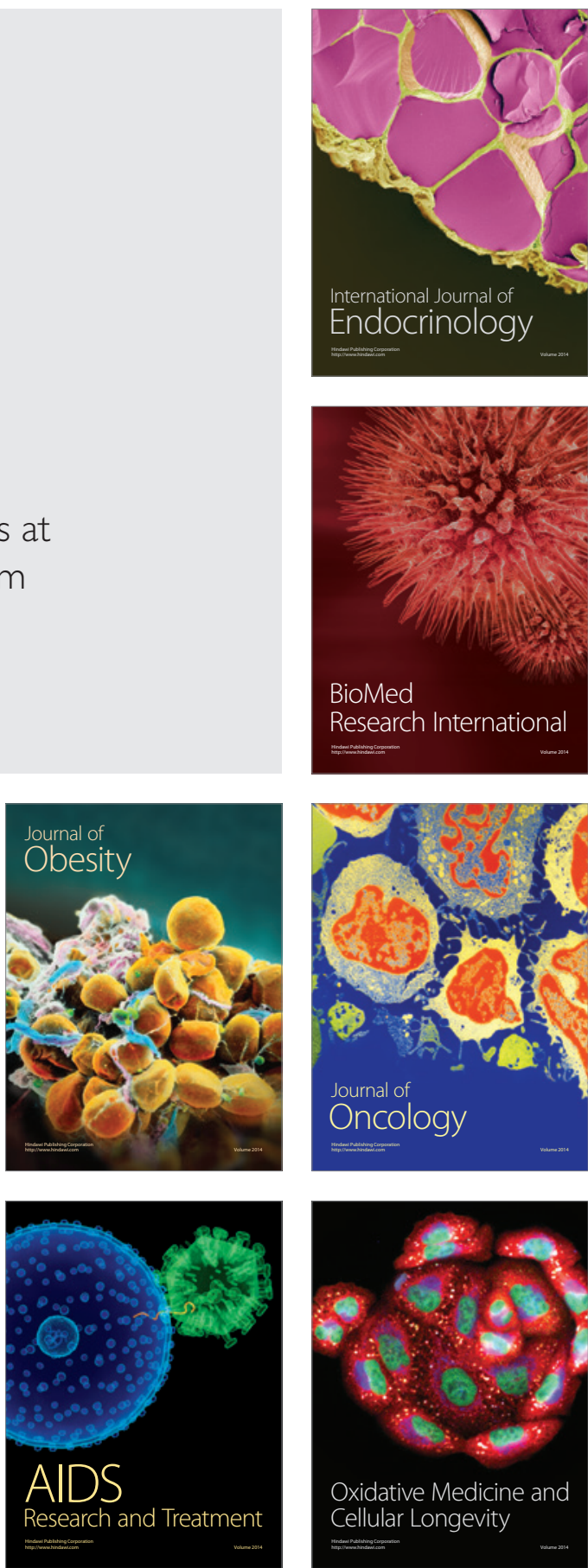